tonitis. It died on the fifth day after operation. Necropsy disclosed general peritonitis from leakage, with no effort at repair.

These two cases illustrate dangers of the operation which cannot be overestimated, and show that even though one has had some experience in this work, fatal errors in technic may and do occur.

Of the seven fatal cases, so far as we know, only one had had careful medical attention and treatment from the beginning of the symptoms; most of the patients had made an occasional visit to the doctor or dispensary and a few had been seen by specialists in consultation, whereas, of the fifteen that recovered, nine were private patients, all of whom had been treated skilfully by competent men from the onset of symptoms, and had been seen in consultation by either Drs. Holt, Kimball, Kerley or La Fetra. These cases came to operation after a reasonable time had been spent in attempting to control the symptoms by medical means; the patients were still in good condition, and as a result made practically uninterrupted recoveries. Two patients died after discharge from the hospital, from causes in no way connected with the operation. Patient 2 was discharged twelve days after operation, having gained 4 ounces and was brought back four days later with an acute gastro-enteritis from which it died. Complete necropsy showed the wound in perfect condition and the cause of death to be acute enteritis. In the other fatal case (No. 14), the patient was discharged July 31, 1913, and died Nov. 2, 1913, of laryngeal diphtheria. The condition had been splendid and just before death the baby weighed 15 pounds. Partial necropsy showed that the tumor, which was rather small, but very hard at operation, persisted.

Careful examination of the stomachs removed at necropsy from the patients dying after operation showed that the pyloric tumors were almost exactly of the same size and consistency as they had been at the operating-table, varying in size from the terminal phalanx of the ring-finger to that of the thumb and having the consistency of cartilage. They were formed entirely by the hypertrophied circular muscle-fibers of the pylorus. The mucous membrane lay in longitudinal folds in the lumen. It was much thickened and arranged in a more or less spiral manner.

In view of the experience gathered from the observation of these cases, I believe I am justified in offering the following conclusions:

1. Hypertrophic pyloric stenosis is congenital to the extent that there is an increase in the thickness of the circular muscle-fibers at the pylorus. The presence of this thickened muscle-fiber reduces the lumen of the pylorus, and therefore the stomach, in order to empty itself, contracts more forcibly than normal. This abnormal contraction soon causes the mucous membratle to become thickened and edematous, and assume a more or less spiral arrangement as it passes through the narrowed pyloric channel of from $\mathrm{r} / 2$ to $3 / 4 \mathrm{inch}$. The result is a valvular action which gradually procluces complete closure of the pylorus. The question as to whether or not the pylorus will admit a probe or catheter at operation or necropsy is of little consequence when weighed against the clinical evidence of complete obstruction.

2. That there is sufficient time between the onset of symptoms and the appearance of the signs of complete obstruction, for careful observation and the carrying out of any medical measures likely to prove of benefit, there can be no doubt, provided, of course, that the early symptoms have been properly interpreted. The fear, however, that the condition may have existed longer than has been suspected, and that the vitality of the baby is not so good as appearances would lead us to believe, makes me feel that operation is indicated in every case of hypertrophic stenosis as soon as the diagnosis is made. Should depression or early evidence of shock be -present, immediate operation is demanded.

3. The babies coming to operation in good condition suffer little or no shock; their convalescence is straightforward, and they are at once restored to normal health. $\mathrm{My}$ experience in this respect corresponds with that of other operators.

I cannot close this paper without referring to the wonderful results obtained by Drs. Scudder of Boston and Richter of Chicago in the operative treatment of pyloric stenosis. The former has recently reported a series of seventeen cases with four deaths and the latter has a record of twenty-two cases with three deaths.

37 West Seventy-First Street.

\section{AN INTERNAL ALEXANDER OPERATION *}

\section{HENRY T. BYFORD, M.D. CHICAGO}

Retroversion of the uterus may be associated with general abdominal and pelvic visceroptosis, or it may be due to impaired function of one or more of the so-called uterine supports. In the latter case there is usually a secondary impairment of function of the remaining supports which are, of course, thrown out of normal action in consequence of the abnormal position of the uterus. An operation to relieve such a deformity does not necessarily have to attack all of the overstretched or relaxed structures, for, when the equilibrium of the uterus is restored and maintained for a certain length of time, those supporting structures which are only slightly deficient or are secondarily disabled may often be depended on to resume function. In limiting myself to the discussion of this class of cases, I am assuming that there is no defect in the pelvic floor, or that such defect has been remedied.

The operation which has proved most satisfactory under these conditions is the Alexander operation. It is one of the simplest of operations, consisting merely of drawing the round ligaments out through their normal channels, the inguinal canals, until the fundus is pulled to its normal position over the empty urinary bladder, and of reattaching them so as to eliminate their apparent excess in length. The part played by these ligaments in connection with retroversion and the Alexander operation is about as follows: The round ligaments are relaxed when the bladder is empty, or nearly so, although they may be supposed to contract more or less irregularly and effectually when stimulated by the shiftings or motions of the uterus accompanying general physical exercise, forced respiration, coughing defecation, etc. As the bladder fills and pushes the fundus uteri upward, the ligaments are

* Read before the thirty-ninth meeting of the American Gynecological Society, May 19, 1914. 
put on the stretch and pull gently downward and forward until the emptying of the bladder enables them to contract and replace the fundus. When we consider the fact that they consist largely of muscular fibers and are apt to be temporarily exhausted by steady prolonged resistance, we can realize how retroversion may occur in a person with relaxed pelvic connective tissue who habitually allows the bladder and rectum to become and remain distended. I can conceive how overstretching of the round ligaments can thus become the chief defect, and the relaxation of the tissues behind the cervix may be of secondary importance, and how a restoration to function of the round ligament may become the rational and effective treatment.

When, however, we shorten them, they are not only restored to action, but also made to compensate, to a certain extent, for the relaxation of the cervical supports. We accomplish this by pulling the ligaments taut so that they will not allow the bladder to push the fundus quite so far back. Now if we did nothing more than this there is no doubt but the displacement would frequently recur as a result of the inefficiency of unaided muscle as a steady support, and the delicacy of the connective-tissue structure of the ligaments.

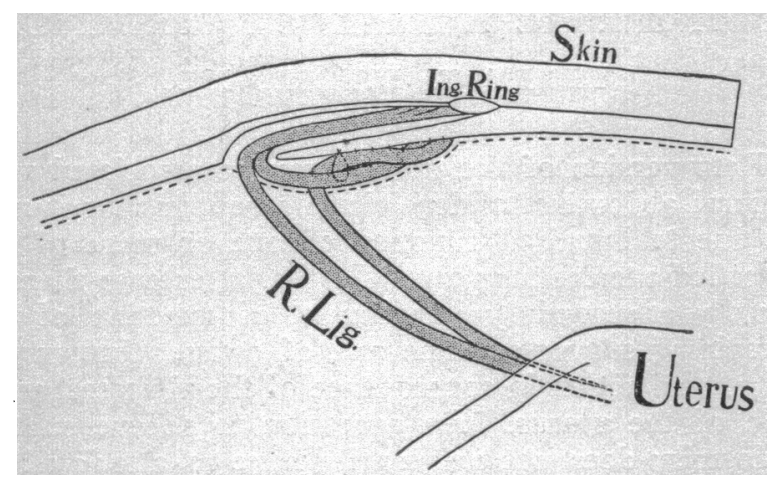

Showing the direction of the normal ligament into the internal inguinal ring, and the shortened ligament folded and attached to the abdominal

Hence to render a recurrence less likely we must warn the patient against holding the urine very long, and provide some means of enabling the overstretched sacro-uterine ligaments to contract and regain some of their lost efficiency. A small pessary-one large enough to keep the cervix from coming too far forward during abdominal pressure, and to help overcome the contraction of the vesicovaginal septum due to the prolonged forward dislocation of the cervix, yet small enough to allow of some play of the cervix and thus of some action of the cervical supports - will ustually prove a valuable aid to the Alexander operation, and render unnecessary any operative treatment of the sacro-uterine ligaments. On the removal of this small pessary after several months, the cervix is usually found to remain appreciably farther back in the pelvis than it did right after the operation without a pessary.

When the peritoneal cavity is opened by a low median incision for complications of retroversion or for other conditions, we can usually save time by shortening the ligaments through the incision instead of by the Alexander method. But all of the intraperitoneal methods that have been recommended, as far as I have been able to determine, have either proved unreliable or have been associated with objectionable features with regard to their conception or to the resulting complications. The simple folding of the round ligaments is unreliable. Suturing of the folds to the uterus usually produces peritoneal exudates which are often followed by pathologic conditions of the organ with disturbance of its functions. Suturing of the folds to other parts either is unreliable or produces rigid exudates. The passing of the severed ligament, or a loop of it, through the abdominal wall, substitutes a fixed displacement for a changeable one. It is more like carpenter-work than surgery, and is admissible only when circumstances do not allow us to do better. I have not the time here for an enumeration of the objections to each of the numerous methods that have been recommended in our current literature, but they may be classified under the headings, unreliability, adhesions, pathologic exudates, abnormal position, restricted mobility, intraperitoneal traumatism, and fundamental errors in conception of objects to be accomplished.

The Alexander operation is freer from these objections than any of the intraperitoneal operative procedures, and in some cases, such as in fat nulliparas, I close the abdominal incision and perform it rather than enlarge the incision and subject the patient to the additional morbidity that so often follows the intraperitoneal methods under unfavorable conditions. The method I now employ, however, avoids intraperitoneal complications and leaves the parts in about the same favorable condition as the Alexander operation. It consists in making a fold near the distal end of the ligament and attaching it intra-abdominally, but extraperitoneally near the internal inguinal ring. Its execution, however, is so simple that a large incision is unnecessary, and it requires much less time, and occasions less trauma than an added Alexander operation. The steps are about as follows:

After the intra-abdominal work for other conditions has been completed, the round ligament is grasped by forceps and pulled out of the inguinal canal until it becomes taut. It is then transfixed by a needle threaded with fine chromic catgut at a point about a centimeter from the internal ring and again about 3 or $4 \mathrm{~cm}$. from the uterine end, and the thread tied so as to make a loop of ligament. The sides of the loop are then sutured with fine chromic or plain catgut forming a sort of double cord. The same is done on the other side and the parts palpated to determine, while there is still time to correct an error in judgment, whether the amount of shortening has been sufficient or excessive.

The peritoneum is next separated freely from the abdominal wall low down on one side of the incision as far laterally as the internal inguinal ring. There is practically no resulting bleeding, and by inserting a short retractor between the peritoneum and rectus muscle one can raise the abdominal wall and do the subsequent work by the aid of sight and touch. With a slender, slightly curved pair of snap-forceps, a puncture from without inward is made in the peritoneal membrane near the internal inguinal ring, and the end of the loop of ligament grasped and pulled through the puncture until the sutured portion of the ligament is all extraperitoneal.

The loop is now given a half twist on itself and, with a curved needle and permanent suture, its base is sutured to the inner surface of the abdominal wall 
as near the internal ring as possible without danger of puncturing the epigastric artery. The loop of ligament, which is practically a short double cord, can now be horizontally sutured along the abdominal wall with as many or as few absorbable sutures as may seem advisable for the production of adequate adhesions. As the suture that makes the loop is a centimeter away from the internal ring and also from the permanent attachment suture, there is a little play of the ligament at the internal ring and some elastic traction exerted by the normal attachments in the inguinal canal. This play of the ligament prevents the muscular atrophy that takes place in it when it is drawn through the abdominal walls so as to suspend the uterus rigidly.

After the operation has been finished on both sides, the slight oozing of blood that may have taken place between the separated peritoneum and abdominal walls will have ceased and the intra-abdominal pressure will cause a firm coaptation of the raw surfaces as soon as the incision is closed. If the loop of ligament has been drawn carefully through a small peritoneal puncture, the peritoneal cavity will be completely shut off from the space in which the ligament is sutured and this space can be safely disinfected with an efficient germicide. I usually sponge it out with a $1: 2,000$ solution of mercuric chlorid in case there has been any chance for infection. I have never seen any bad results from separating the suprapubic peritoneum from the abdominal for this purpose.

Should the permanent suture give way and the ligament separate from its attachment to the abdominal wall, the loop will still be held by its subperitoneal embedded attachment, for it cannot suddenly slip through the peritoneal puncture after having grown into it. Nor are the sides of the loop of round ligament very liable to be torn apart after having grown into the subperitoneal connective tissue. Hence the chances of failure are not so great as they would be if the success depended only on attaching the ligament intraperitoneally to the inner surface of the abdominal parietes.

As the only intraperitoneal part of this method consists in making the small loop of ligament and suturing its sides together, and as this loop is drawn through a puncture until it entirely disappears from the peritoneal cavity, and as the ligament pulls from the neighborhood of the internal inguinal ring, I feel justified in saying that the intraperitoneal conditions after the operation, as far as the shortening of the ligaments is concerned, are the same as after an Alexander operation.

32 North State Street.

Home-Project Agricultural Instruction. - Massachusett has a law providing for agricultural courses in all stateaided schools. Pupils in the agriculturai courses in the schools are required to carry out a certain amount of agricultural work at their homes, under supervision of the schoolteachers during the summer vacation. A number of these schools have been established, and much enthusiasm has been shown by the pupils, and great interest is taken by the parents in these projects. The interest of the pupils in school work is thus kept up beyond the age of 14 , when many of them would otherwise leave school. It makes for good citizenship and should have great possibilities for furthering public health and sanitation. A report covering this work has been issued by the United States. Bureau of Education as Bulletin, 1914, No. 8.

\section{THE TREATMENT OF TETANUS BY ANTITOXIN}

\section{ERNEST E. IRONS, M.D.} CHICAGO

The efficacy of antitetanic serum in the treatment of developed tetanus has been questioned by many experienced clinicians, who have regarded the recoveries in occasional cases of tetanus under large doses of serum as fortuitous; and if one may judge from the lukewarm advocacy of serum in most text-books, the general impression of the profession is that but little can be accomplished by its use. A study of the mortality statistics of most hospitals shows that this skepticism too often has been well founded. Opposed to this pessimistic view are the reports of a number of small groups of carefully observed cases, such as those of Ashhurst and John, in which the evidence seems strong that antitoxin deserved large credit for the recovery of some of the patients, and that the mode of administration of the remedy is a very important element in its success.

The collection of the 225 reports of cases here analyzed was undertaken with the object of ascertaining, in the first place, what results are being obtained in this country with antitetanic serum in the treatment of tetanus, and, in the second place, whether the failures in some cases may not be ascribed to the faulty and inefficient method of giving the serum. Statistical studies of case-reports collected from the literature are necessarily subject to error, and particularly in tetanus, such tabulations have led to conclusions favorable to serum treatment to a degree unwarranted by the actual facts.

With one or two possible exceptions, none of the cases in this series have been reported, and all were obtained by personal correspondence from hospital or private records, for the years 1907 to $1913 .^{1}$

TABLE 1.-MORTALITY OF THE SEVERAL CLASSES OF CASES

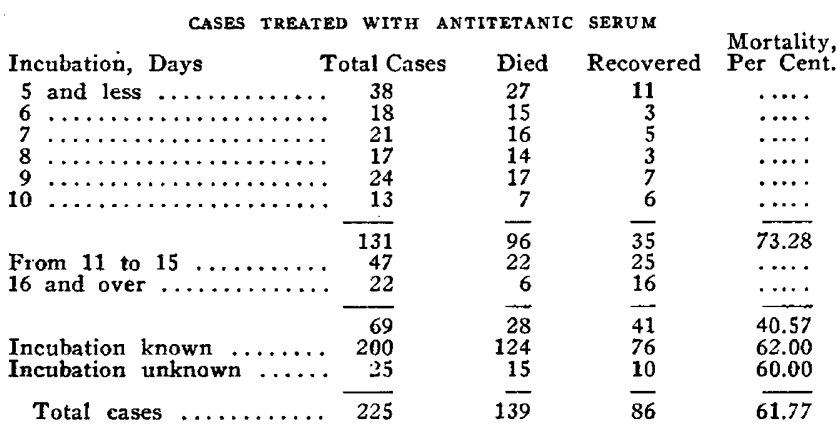

\begin{tabular}{|c|c|c|c|c|c|c|}
\hline & CASES $T$ & ATED & WITHOUT & SERUM & & \\
\hline $\begin{array}{l}10 \text { and less } \\
\text { Over } 10 \ldots \\
\text { Unknown } \ldots\end{array}$ & 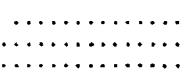 & $\begin{array}{r}12 \\
4 \\
5\end{array}$ & $\begin{array}{r}11 \\
2 \\
5\end{array}$ & & $\begin{array}{l}1 \\
2 \\
0\end{array}$ & $\begin{array}{l}\ldots \ldots \\
\ldots \ldots \\
\ldots \ldots\end{array}$ \\
\hline Total case & & 21 & 18 & & 3 & 85.7 \\
\hline
\end{tabular}

Table 1 summarizes the mortality of the several classes of cases. The mortality in all cases receiving

1. All cases reported by any one observer are included, so far as the completeness of the data permit. The data of 80 cases were obtained by me directly from hospital records. About 20 cases occurred in the private practice of physicians. The remainder were hospital cases; treated for the most part in large metropolitan haspitals, including Bellevue, Massachusetts General, Boston City, Buffalo, Philadelphia General, Royal Victoria and Cook County, Wesley, Presbyterian and Michael Reese in Chicago. To those who have so kindly assisted in the collection of cases my best thanks are extended. A fuller acknowledgment will be made in the complete paper in the Journal of Infectious Biseases. 